\title{
Identification of a displaced glottis using vocalization
}

\author{
Yuko Ono $\cdot$ Yoshinobu Abe $\cdot$ Kazuaki Shinohara
}

Received: 11 June 2014/ Accepted: 4 September 2014/Published online: 26 September 2014

(C) Japanese Society of Anesthesiologists 2014

To the Editor:

A 72-year-old obese male was brought to the hospital after being involved in a home fire. He had inhaled massive hot air and developed severe dyspnea. On admission, he exhibited hoarseness and superficial dermal burns over his entire face. He had labored breathing and orthopnea. Fiberoptic bronchoscopic (FOB) examination revealed severe upper-airway edema. Airway collapse appeared imminent. We attempted awake endotracheal intubation (ETI) with emergency surgical airway (ESA) backup. After the patient was adequately oxygenated, topical anesthesia was applied followed by intravenous midazolam $2 \mathrm{mg}$ and fentanyl $0.1 \mathrm{mg}$. This allowed him to be lightly sedated but still able to communicate. Unfortunately, two direct laryngoscopies failed, and progression of upper-airway edema and low respiratory reserve made FOB intubation impossible. Before performing an ESA, we attempted ETI with the Pentax Airway Scope (AWS). We could elevate the swollen epiglottis directly with the blade of the AWS, but increasing laryngeal edema had displaced the glottis. We then asked the patient to vocalize. Lateral movement and vibration of the vocal cords helped us to identify the glottis, and we achieved ETI with a 7.0-mm endotracheal tube. After discharge, the patient returned to his normal activities.

Unique to our experience was the successful identification of the displaced glottis by having the patient vocalize. This technique may work in other intubation attempts, including FOB insertion and direct laryngoscopy. In this challenging case, the high-quality monitor of the AWS provided a clear image of vocal cord movement, which greatly aided in identifying the glottis. Controlled sedation and analgesia are paramount in achieving this intervention.

In conclusion, the key intervention in this severe inhalation-burn case was having the patient vocalize so that we could identify the displaced glottis. The AWS was useful in facilitating this technique.

Conflict of interest None.
Y. Ono $(\bowtie) \cdot$ Y. Abe

Emergency and Critical Care Medical Center, Fukushima

Medical University Hospital, 1 Hikarigaoka,

Fukushima 960-1295, Japan

e-mail: windmill@fmu.ac.jp

K. Shinohara

Department of Anesthesiology and Critical Care Medicine,

Ohta General Hospital Foundation, Ohta Nishinouch Hospital,

Koriyama, Japan 